\title{
STEADY MASS LOSS ASSOCIATED WITH NOVA OUTBURSTS*
}

\author{
MARIKO KATO \\ Department of Astronomy, Faculty of Science, University of Tokyo, Bunkyo-ku, Tokyo, Japan
}

(Received 6 June, 1983)

\begin{abstract}
The structure of optically thick mass-losing envelopes of white dwarfs are studied in relation to nova outbursts. A sequence of steady mass-loss solutions is constructed for a nova outburst from the maximum photospheric radius to the disappearance. Much of mass of the envelope will be blown out.
\end{abstract}

\section{Nova Outbursts}

Nova is thought to be an outburst phenomena triggered by the nuclear shell flash in the hydrogen-rich envelope of an accreting white dwarf in a close binary system. The onset of shell flash and the initial phase of expansion of envelope in the nova outburst have been studied by various authors (see, e.g., Truran, 1981; Nariai et al., 1980, and references therein). Such time-dependent calculations show that the envelope greatly expands and the velocity of outermost mesh point exceeds the escape velocity. However, the subsequent stages of great expansion have not been calculated yet. Such a extended stage can be treated by steady mass-loss (e.g., Ruggles and Bath, 1979). The present paper concerns a sequence of steady mass-loss solutions for a nova outburst from the maximum expansion to the termination of the mass loss (for details, see Kato, 1983b).

\section{Steady Mass-Loss Solution}

The basic equations governing the structure of the envelopes are the equations of motion, continuity, energy conservation, and energy transfer by diffusion process (Kato, 1983a). The chemical abundances of hydrogen, helium, and heavy elements are assumed to be $X=0.73, Y=0.25$, and $Z=0.02$ by weight, respectively. The mass and the radius of the white dwarf are taken to be $M=1.3 M_{\odot}$ and $R=0.0041 R_{\odot}$.

Some of the solutions obtained are shown in Table I. Here $\dot{M}$ is the mass loss rate. The subscript ph denotes the values at the photosphere. Figure 1 shows the variations in the diffusive luminosity $L_{r}$ and the Eddington luminosity $L_{\mathrm{Ed}}=4 \pi c G M / \kappa$. The short vertical lines and the crosses denote the photosphere and the critical point, respectively. The Eddington luminosity is a local variable and of which dips correspond to the ionization zones of helium and hydrogen. The luminosity becomes super-Eddington there.

\footnotetext{
* Paper presented at the Lembang-Bamberg IAU Colloquium No. 80 on 'Double Stars: Physical Properties and Generic Relations', held at Bandung, Indonesia, 3-7 June, 1983.
} 
TABLE I

Properties of the thermal equilibrium envelopes

\begin{tabular}{llllllll}
\hline Model & $\begin{array}{l}\dot{M} \\
\left(\mathrm{~g} \mathrm{~s}^{-1}\right)\end{array}$ & $\begin{array}{l}\log r_{\mathrm{ph}} \\
(\mathrm{cm})\end{array}$ & $\begin{array}{l}\log T_{\mathrm{ph}} \\
(\mathrm{K})\end{array}$ & $\begin{array}{l}\log v_{\mathrm{ph}} \\
\left(\mathrm{cm} \mathrm{s}^{-1}\right)\end{array}$ & $\begin{array}{l}\log v_{\mathrm{esc}} \\
\left(\mathrm{cm} \mathrm{s}^{-1}\right)\end{array}$ & $\begin{array}{l}L_{\mathrm{ph}} \\
\left(10^{38} \mathrm{erg} \mathrm{s}^{-1}\right)\end{array}$ & $\begin{array}{l}\log \Delta M \\
\left(M_{\odot}\right)\end{array}$ \\
\hline E1 & $8.20(23)$ & 13.18 & 3.78 & 7.41 & 6.53 & 2.13 & -3.59 \\
E2 & $2.05(22)$ & 12.98 & 3.84 & 6.88 & 6.63 & 1.48 & -4.70 \\
E3 & $1.12(22)$ & 12.96 & 3.85 & 6.83 & 6.64 & 1.51 & -4.87 \\
E4 & $2.85(21)$ & 12.84 & 3.92 & 6.64 & 6.70 & 1.58 & -5.22 \\
E5 & $2.08(21)$ & 12.80 & 3.94 & 6.55 & 6.72 & 1.60 & -5.29 \\
E6 & $3.25(20)$ & 11.74 & 4.47 & 6.53 & 7.25 & 1.69 & -5.86 \\
E7 & $7.22(19)$ & 11.47 & 4.61 & 6.39 & 7.38 & 1.75 & -6.18 \\
\hline
\end{tabular}

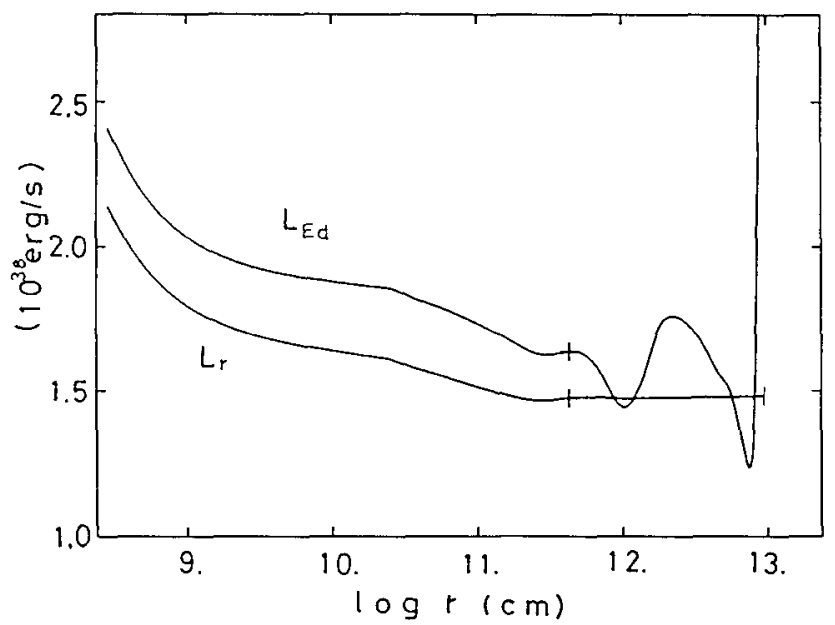

Fig. 1. Distributions of the energy flux by diffusion and the Eddington luminosity $L_{\mathrm{Ed}}=4 \pi c G M / \kappa$.

\section{The Condition of Mass Loss to Occur}

We obtain the solutions only when the diffusive luminosity at the bottom of the envelope $L_{\mathrm{c}}$ is larger than the minimum value of the Eddington luminosity - i.e.,

$$
L_{c}>L_{\mathrm{Ed}, \min } \text {. }
$$

This suggests that the mass loss occurs when the diffusive flux $L_{c}$ is greater than a critical value which is determined by the opacity not at the bottom of the envelope but in the surface region.

When a shell is static and mass loss does not occur, the diffusive luminosity is constant $\left(L_{r}=L_{c}\right)$ except in the nuclear burning region. On the other hand, the Eddington luminosity is a local variable and generally decreases outward. If $L_{c}$ is less than the minimum value of $L_{\mathrm{Ed}}$, mass loss may not occur. But if $L_{c}$ becomes as large as $L_{\mathrm{Ed} \text {, min }}$, mass loss will begin. The luminosity then decreases outward in order to raise 
the matter against the gravity of the white dwarf. Consequently, the luminosity becomes everywhere sub-Eddington in models where mass loss just sets in. In the model where $L_{c}$ is much larger than $L_{\mathrm{Ed} \text {, min }}$, regions of super-Eddington luminosity appear near the surface of the greatly extended envelopes, where the opacity steeply increases in the partial ionization zones of hydrogen and helium.

\section{Nova Outbursts and Steady Mass Loss}

Fiure 2 gives a schematic diagram for the existence of the solutions with mass loss. Because the acceleration of matter occurs in the envelope $\left(R_{\mathrm{WD}}<r_{\mathrm{cr}}<r_{\mathrm{ph}}\right)$, the solutions exist in the region enclosed by two curves $r_{\mathrm{cr}}=r_{\mathrm{ph}}$ and $r_{\mathrm{cr}}=R$. In the lower region to the line $r_{\mathrm{cr}}=r_{\mathrm{ph}}$, static solutions may exist instead (for detail, see Kato, 1983b).

Progress of a nova outburst is schematically explained by using Figure 2. According to the theory of shell flash, the thermal run-away commences with hydrogen ignition, when the mass accumulated on the white dwarf exceeds some critical value. Just after the ignition of the hydrogen burning the envelope begins to expand because the energy generation by nuclear burning is much larger than the outward energy flux. When the envelope expands greatly, the early explosive expansion will settle down to a milder mass-loss phase in a steady state. Such a state is not only dynamically steady but also thermally steady. The locus denoted as thermal equilibrium in Figure 2 describes the thermally steady-state models in which the energy loss is balanced by the energy generation. The envelope will stay on this line for a long time and then it will slowly move along leftward as the mass $\Delta M$ is decreased by mass loss. After the envelope reaches point $B$, the mass-loss will stop and the envelope may settle down to a static structure. It will shrink according to the decrease of $\Delta M$ due to the hydrogen burning and eventually the nuclear burning will be extinguished.

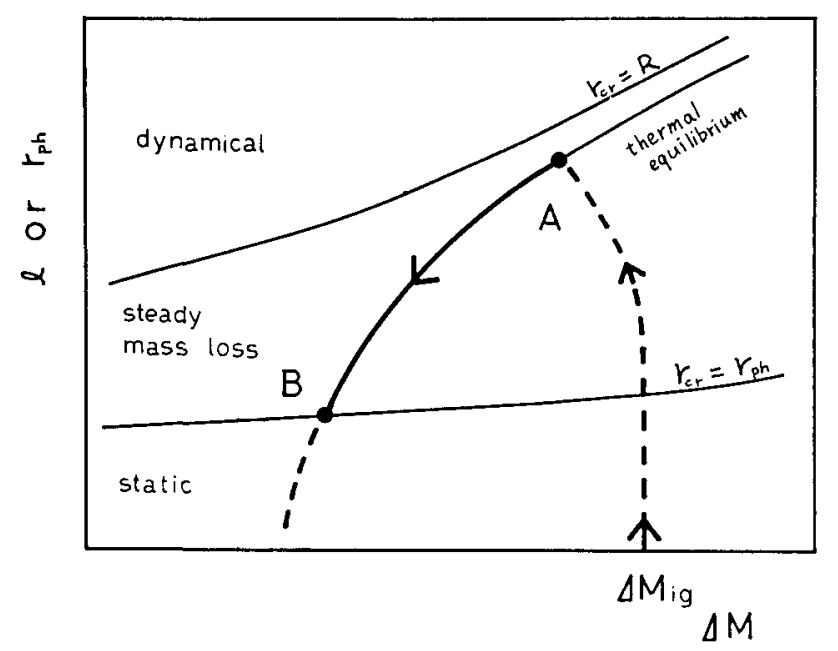

Fig. 2. Schematic diagram for the existence of steady solutions and the evolution of a nova outburst. 
Table I shows a sequence of models corresponds to the line $E$. In the theory of shell flash, the ignition mass $\Delta M_{i g}$ is estimated to be $2 \times 10^{-6} \sim 10^{-4} M_{\odot}$ for $M=1.3 M_{0}$ when the accretion rate is $10^{-7} \sim 10^{-11} M_{\odot} \mathrm{yr}^{-1}$ (Nariai and Nomoto, 1979). The time scale to move leftward on the line $E$, which is estimated by $\Delta M / \dot{M}$, is about $6 \times 10^{6} \mathrm{~s}$ for Model E1 and about $2 \times 10^{7} \mathrm{~s}$ for Model E7.

\section{References}

Kato, M.: 1983a, Publ. Astron. Soc. Japan 35, 33.

Kato, M.: 1983b, Publ. Astron. Soc. Japan 35, in press.

Nariai, K. and Nomoto, K.: 1979, Proceedings of Fourth Annual Workshop on Dwarf Novae and Other Cataclysmic Variables, Rochester, p. 525.

Nariai, K., Nomoto, K., and Sugimoto, D.: 1980, Publ. Astron. Soc. Japan 32, 473.

Ruggles, C. L. N. and Bath, G. T.: 1979, Astron. Astrophys. 80, 97.

Truran, J. W.: 1981, in D. Wilkinson (ed.), Progress in Particle and Nuclear Physics, Vol. 6, Pergamon Press, Oxford. 